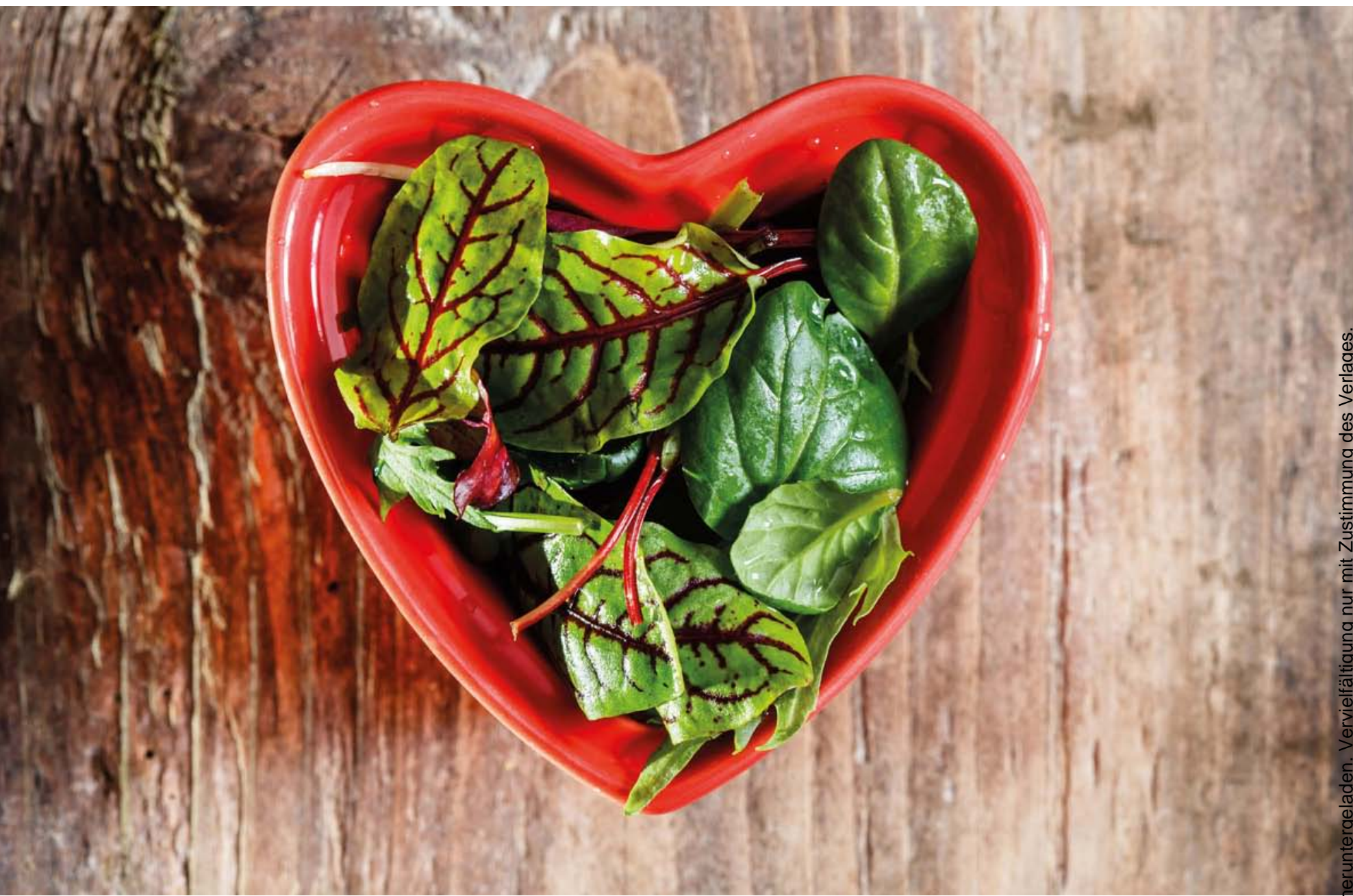

\title{
Richtig essen allein reicht nicht
}

\author{
Welche ERNÄHRUNGSFORM und welche Nährstoffe bei KHK sind richtig und wichtig? \\ Volker Schmiedel
}

ES IST ERSTAUNLICH. Veganer ernähren sich ballaststoffreicher als Karnivoren (Fleischesser). Sie nehmen mit der Nahrung mehr antioxidative Vitamine und sekundäre Pflanzenstoffe auf. Im Vergleich leiden sie seltener an Diabetes mellitus, Bluthochdruck oder Übergewicht. Doch das Risiko, an einem Herzinfarkt zu sterben, ist für einen Veganer um $40 \%$ größer als für einen Fleischesser [1].

Liest man dieses Studienergebnis, ist die Frage berechtigt: Sind unsere Ernährungstheorien richtig, die besagen: kaum Fleisch, viel pflanzliche Nahrung? Oder gibt es evtl. wichtigere Einflussfaktoren?

\section{Die richtige Ernährung: Nur ein Mythos?}

Auf die Frage nach der richtigen Ernährung die richtige Antwort zu geben - es ist fast unmöglich. Beinahe grenzenlos scheinen wir zwischen Ernährungsformen wählen zu können von denen jede vorgibt, die gesündeste überhaupt zu sein. Ist also die Instinktotherapie die richtige? Oder ist es besser, sich ausschließlich von Rohkost oder gemäß der Vollwertkost nach Bruker zu ernähren? Oder High Carb? Oder vielleicht Low Carb? Sollte man sich an der in anderen Ländern üblichen Ernährungsweise orientieren? Die mediterrane Diät ist dann noch die, die uns im wahrsten Wortsinn am nächsten liegt. Ayurvedische Kost ist aber auch sehr beliebt, ebenso die Ernährung nach TCM. Die vegetarische Kost, ganz gleich ob aus ethischen oder gesundheitlichen Gründen gewählt, bietet ebenfalls viele Möglichkeiten: Manche Vegetarier sind moderat und entscheiden sich für ein Dasein als Flexitarier, was bedeutet, weniger als einmal in der Woche Fleisch zu essen. Es gibt Ovo-Lacto-Vegetarier, die Eier und Milchprodukte zu sich nehmen; Pesco-Vegetarier, die nicht von Fischen lassen. Nur Veganer verzichten auf sämtliche tierische Nahrung. Doch auch das lässt sich steigern: Neben Rohkost-Veganern gibt es Fruganer. Sie essen nicht die ganze, leben- 
de Pflanze, sondern nur deren Samen und Früchte. Was also ist die richtige Ernährung?

Dieser Beitrag stellt „nur“ die Frage nach der Ernährungsform, die bezogen auf den Schutz der Koronargefäße die richtige ist, sprich: Welche Kostform ist die geeignete, um Verengungen bzw. Infarkte der Herzkranzgefäße zu vermeiden? Die Antwort ist daher vergleichsweise einfach.

\section{Pflanzenkost schützt das Herz}

Es gibt seit vielen Jahren Hinweise, dass vegetarische Kostformen sich am wenigsten nachteilig auf die Koronararterien auswirken. Der geringe Gehalt an gesättigten Fettsäuren, Cholesterin bzw. der niedrige Gesamtfettgehalt der pflanzlichen Ernährung wird meist als Begründung angeführt. Doch fällt der hohe Gehalt an Ballaststoffen, antioxidativen Vitaminen und sekundären Pflanzeninhaltsstoffen stärker ins Gewicht. Alles Schutzfaktoren für die Gefäße.

Kritiker bemängeln jedoch methodologische Probleme bei den Ernährungsstudien: Es sind fast ausschließlich epidemiologische Studien, bei denen nur die Auswirkungen der Einflussfaktoren auf Morbidität oder Mortalität gemessen werden, die der Mensch selbst gewählt hat - in diesem Fall die Ernährung. Andere Einflussfaktoren bleiben unberücksichtigt - und die sind leider nicht zufällig verteilt: Vegetarier trinken durchschnittlich weniger Alkohol, rauchen seltener, bewegen sich mehr, haben eine bessere Bildung und ein höheres Einkommen. All das korreliert mit einer geringen Morbidität bzw. Mortalität bei Koronarer Herzkrankheit (KHK).

Was ist nun bedeutsamer: Sind es diese psychosozialen oder doch eher die diätetischen Faktoren?

\section{Mediterrane Kost reduziert Herzrisiko}

Epidemiologische Studien können Hinweise liefern auf Schutzoder Risikofaktoren, jedoch keine Beweise. Das Nonplusultra unter den klinischen Studien, die kontrollierte, randomisierte Doppelblindstudie, eignet sich aus naheliegenden Gründen nicht. Kontrollierte, randomisierte Studien sind in der Ernährungsforschung aber immerhin durchführbar, wenn auch nicht ganz einfach: Die Teilnehmer müssen bereit sein, sich über längere Zeit der einen oder anderen Ernährungsform gemäß zu ernähren, was aus verständlichen, menschlichen Gründen mitunter an die Grenzen des Machbaren stößt. Es sind jedoch erfolgreich Anstrengungen unternommen worden. Eine der bekanntesten Studien ist die Lyon Diet Heart Study. Randomisiert wurden 2 Gruppen gebildet. Die Teilnehmer in einer Gruppe ernährten sich nach der sog. westlichen Diät, also wie in Nordfrankreich und Mitteleuropa üblich mit einem hohen Fleisch- und Milchproduktanteil. Die Teilnehmer der 2. Gruppe aßen bevorzugt nach den Regeln der mediterranen Diät.

Bei pharmakologischen Studien kann man voraussetzen, dass das Medikament bzw. Placebo eingenommen wird, die Compliance lässt sich durch Zählen der übrig gebliebenen Tab-

\section{KURZ GEFASST}

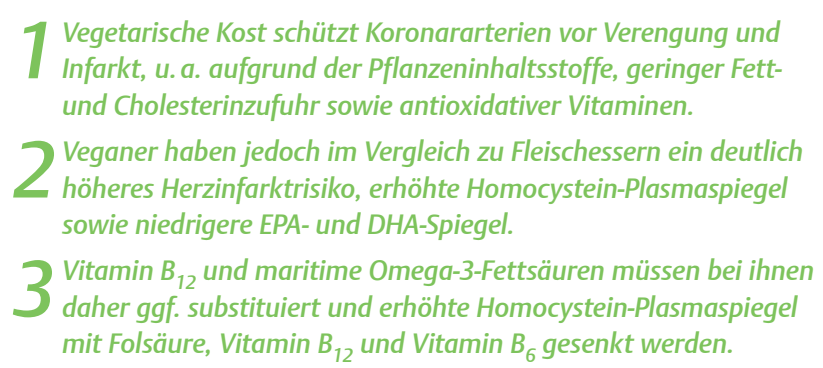

letten bzw. Bestimmung des Medikamentenspiegels im Blut sogar überprüfen. Bei einer Ernährungsstudie kann man dagegen nie davon ausgehen, dass die Regeln zu 100\% befolgt werden. So werden sehr wahrscheinlich Studienteilnehmer der westlichen Diätgruppe mal an einem Salat geknabbert und welche der mediterranen auch einmal ein Schweineschnitzel gegessen haben. Anhand geführter Ernährungsprotokolle konnte jedoch eine weitgehende Einhaltung der Diätvorschriften verifiziert werden.

\section{Verblüffende Ergebnisse}

Die Wissenschaftler fanden bei der Nahrungsanalyse heraus, dass die Teilnehmer der Gruppe, die sich nach der mediterranen Diät ernährte, weniger Kalorien, Gesamtfett und Cholesterin zu sich nahmen, dafür aber mehr ungesättigte Fettsäuren, Ölsäure (aus Oliven oder Rapsöl), Omega-3-Fettsäuren (aus Leinöl oder Fischen) und Ballaststoffe.

Innerhalb der durchschnittlichen 4 Beobachtungsjahre kam es bei den insgesamt etwa 700 Studienteilnehmern zu fast 300 Ereignissen wie Herzinfarkt bzw. Krankenhauseinweisung. Eine beachtliche Anzahl für die kurze Zeit. Da stellt sich die Frage: Gab es Unterschiede zwischen den beiden Gruppen?

In der Tat: In der Teilnehmergruppe, die sich gemäß der mediterranen Diät ernährte, war das Herztod- und Herzinfarktrisiko 77\% niedriger als in der Vergleichsgruppe, das Risiko für Angina-pectoris-Anfälle, Schlaganfälle, Herzinsuffizienz oder Embolie 70\% niedriger. Die Zahl der Krankenhauseinweisungen war um $49 \%$ geringer. Raucher hatten hingegen „nur“ $50 \%$ mehr Herztodesfälle oder -infarkte als Nichtraucher, Teilnehmer mit hohem Blutcholesterin „nur“ $31 \%$ mehr.

\section{Der schützende Einfluss der mediterranen Diät ist der Lyon Diet Heart Study zufolge stärker als der schädigende Effekt des Rauchens bzw. Serumcholesterins.}




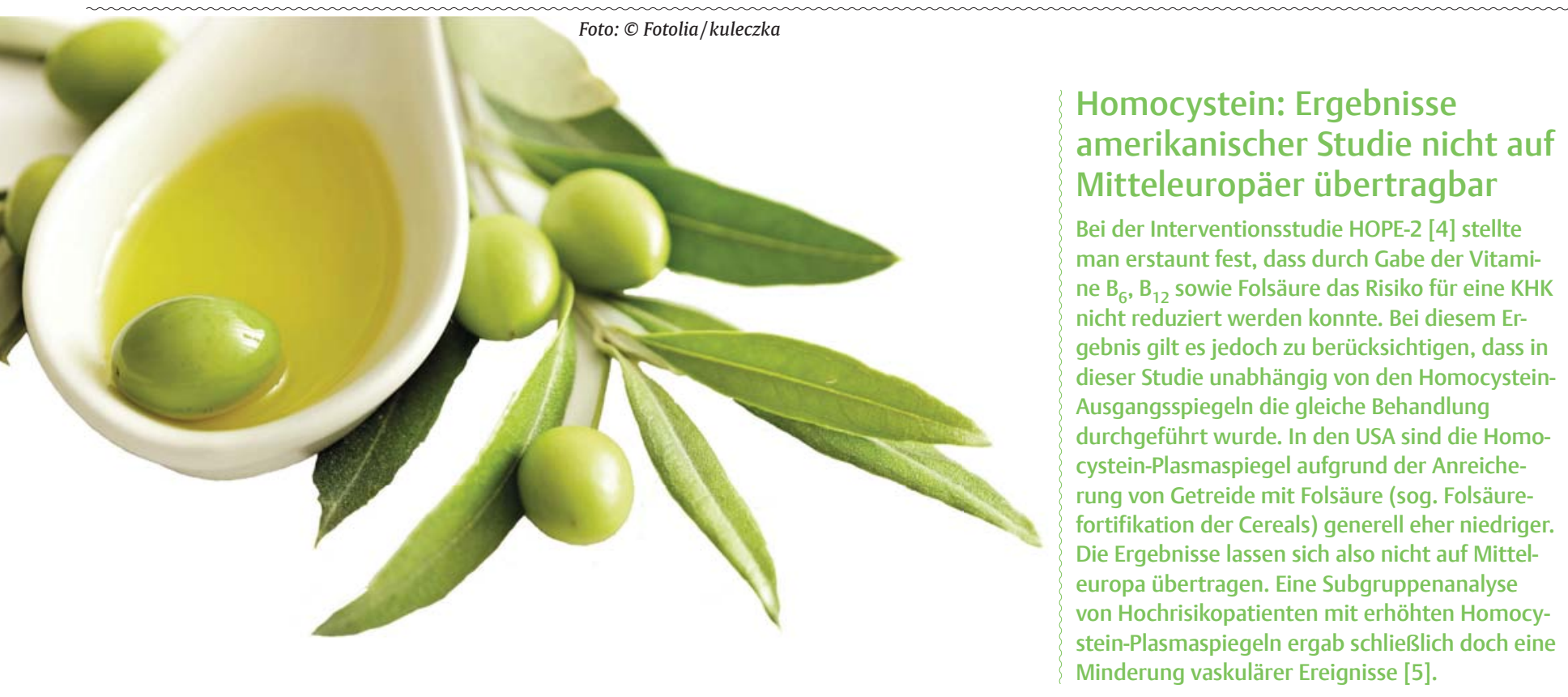

\section{Was wir von 7-Tage-Adventisten lernen können}

Warum aber haben Veganer ein, wie eingangs erwähnt, im Vergleich zu Fleischessern fast $40 \%$ höheres Herzinfarktrisiko?

Das erhöhte Risiko wurde in der sog. 7-Tage-AdventistenStudie 2 festgestellt [1]. Die 73308 amerikanischen 7-Tage-Adventisten sind, was die sozioökonomischen Parameter betrifft, im Vergleich zur Normalbevölkerung eine relativ homogene Klientel. Es gibt innerhalb dieser Religionsgruppe sehr viele Vegetarier unterschiedlicher Ausprägung, z. B. Flexitarier, Ovo-LactoVegetarier, Pesco-Vegetarier und Veganer.

Die Gesamtmortalität war bei den Fleischessern im Vergleich zu den Vegetariern um 12\% höher. Die Veganer hatten jedoch im Vergleich zu den Karnivoren ein um 39\% erhöhtes Sterberisiko, die Pesco-Vegetarier dagegen ein fast halbiertes (-49\%). Das Sterberisiko der Ovo-Lacto-Vegetarier bzw. Flexitarier unterschied sich statistisch nicht von dem der Karnivoren. Der Unterschied zwischen Veganern und Fischessern: beinahe ein um das 2,7-fach erhöhte Risiko bezüglich der Herzsterblichkeit!

Wie sind diese immensen Unterschiede zu erklären? Es gibt dafür bisher nur 2 plausible Hypothesen.

\section{Die Homocystein-Hypothese}

Veganer haben deutlich erhöhte Homocystein-Plasmaspiegel bei gleichzeitig sehr niedrigen Eicosapentaensäure- und Docosahexaensäure-Spiegeln (Eicosapentaensäure [EPA] und Docosahexaensäure [DHA] sind maritime Omega-3-Fettsäuren). In epidemiologischen Studien fanden sich Hinweise auf verstärkte Arteriosklerose- und Krebsinzidenzen, wenn der Homocysteinspiegel im Plasma erhöht ist. In einer norwegischen Studie mit fast 5000 älteren Teilnehmern wurde das Mortalitätsrisiko in Abhängigkeit des Homocysteinspiegels gemessen: Sowohl bei der kardiovaskulären als auch bei der nicht kardiovaskulär bedingten Sterblichkeit (überwiegend Krebs) gab es relevante und signifikante Anstiege bei höheren Homocysteinspiegeln.

Die nicht proteinogene Aminosäure Homocystein, die im Zwischenstoffwechsel entsteht, wird rasch abgebaut, wenn aus- reichend Folsäure, Vitamin $B_{6}$ und $B_{12}$ im Körper vorhanden sind. Herrmann et al. [6] fanden bei Veganern mit 12,8 mmol/l erhöhte Homocystein-Plasmaspiegel, bei Vegetariern betrugen sie $10,6 \mathrm{mmol} / 1$ und bei Fleischessern $8,8 \mathrm{mmol} / 1$, was eindeutig mit den Vitamin $\mathrm{B}_{12}$-Spiegeln korreliert.

\section{Merke: Es besteht bei Veganern nach einigen Jahren ohne Substitution immer ein Vitamin $B_{12}$-Mangel.}

\section{Die Omega-3-Fettsäuren-Hypothese}

Die andere Erklärung für die Risikoerhöhung bei Veganern: Omega-3-Fettsäuren. Trotz geringer Zufuhr der tierischen Omega-6-Fettsäure Arachidonsäure und oft vermehrter Aufnahme der pflanzlichen Omega-3-Fettsäure Alpha-Linolensäure ist der Arachidonsäuregehalt im Blut von Veganern häufig höher als der von Karnivoren. Der Grund: Arachidonsäure wird aus Linolsäure synthetisiert, die Veganer meist in hoher Menge konsumieren. Die Blutspiegel der Omega-3-Fettsäuren EPA und DHA sind dagegen - verglichen mit denen von Normalköstlern - drastisch niedriger. Denn die Syntheserate von EPA aus AlphaLinolensäure liegt bei ihnen unter 10\% (eigene Erfahrungen aus mehr als 500 Fettsäureanalysen).

Omega-3-Fettsäuren schützen vor kardiovaskulären Ereignissen, mehrere epidemiologische und interventionelle Studien belegen das. Das Risiko für einen plötzlichen Herztod ist bei einem Omega-3-Index (Summe von EPA und DHA in Relation zu allen Fettsäuren) von über $8 \%$ um $90 \%$ geringer als bei einem Index von unter 4 [7].

Die bisher größte und bekannteste Omega-3-Studie ist die GISSI-Studie [8]: Mehr als 11000 Patienten mit Z.n. Herzinfarkt erhielten für 3,5 Jahre entweder 850 mg EPA/DHA oder Placebos. In der Verumgruppe sank die Gesamtmortalität um $20 \%$ und das Risiko am plötzlichen Herztod zu sterben um $45 \%$. 


\section{Fazit}

Es gibt überzeugende Hinweise für präventive Effekte vegetarisch orientierter Kostformen bei KHK, zu denen auch eine mediterrane Ernährung zählt. Wer sich streng vegetarisch oder auch vegan ernährt, sollte jedoch unbedingt Vitamin $B_{12}$ und maritime Omega-3-Fettsäuren substituieren. Im Zweifel sollten Messungen der Homocystein-Plasma- sowie der Fettsäurespiegel erfolgen. Bei einer Erhöhung des Homocysteinspiegels ist eine Therapie mit Folsäure, Vitamin $B_{6}$ und $B_{12} z u$ empfehlen und ein Homocystein-Wert von unter $10 \mathrm{mmol} / \mathrm{l}$ anzustreben. Hinsichtlich des Omega-3-Indexes sollte ein Wert von über 8\% erzielt werden (v.a. in der sekundären Prävention), wofür meist eine Tagesdosis von $2 \mathrm{~g}$ EPA/DHA erforderlich ist. Diese Menge ist z.B. in $100 \mathrm{~g}$ Hering, $300 \mathrm{~g}$ Makrele, 1 EL Fischöl oder 12 konventionellen $500 \mathrm{mg}$-Fischölkapseln enthalten (entsprechend weniger bei größeren und / oder aufkonzentrierten Kapseln). Bei fischarmer Ernährung (weniger als 1 Fischmahlzeit pro Woche) werden sogar 3-4g reine Omega-3-Fettsäuren benötigt. =

Dieser Artikel ist online zu finden:

http://dx.doi.org/10.1055/s-0036-1582148

\section{Verwendete Literatur}

[1] Orlich M], Singh PN, Sabaté J et al. Vegetarian Dietary Patterns and Mortality in Adventist Health Study 2. JAMA Intern Med. 2013; 173(13): $1230-1238$

[2] De Lorgeril M, Salen P, Martin JL et al. Mediterranean diet, traditional risk factors, and the rate of cardiovascular complications after myocardial infarction: final report of the Lyon Diet Heart Study. Circulation 1999; 99: 779-785

[3] Vollset SE, Refsum H, Tverdal A et al. Plasma total homocysteine and cardiovascular and noncardiovascular mortality: the Hordaland Homocysteine Study. Am J Clin Nutr 2001; 74(1): 130-136

[4] Lonn E, Yusuf S, Arnold M] et al. Homocysteine lowering with folic acid and B vitamins in vascular disease. N Engl J Med. 2006; 354(15): 15671577
[5] Saposnik G, Ray JG, Sheridan P et al. Heart Outcomes Prevention Evaluation 2 Investigators. Homocysteine-lowering therapy and stroke risk, severity, and disability: additional findings from the HOPE 2 trial. Stroke 2009; 40(4): 1365-1372

[6] Herrmann W, Schorr H, Obeid R, Geisel J. Vitamin B-12 status, particularly holotranscobalamin II and methylmalonic acid concentrations, and hyperhomocysteinemia in vegetarians. Am J Clin Nutr 2003; 78: 131-136

[7] Harris WS. Omega-3 fetty acids and cardiovascular disease: A case for omega-3 index as a new risk factor. Pharmacologocal Research 2007; 55 : 217-223

[8] GISSI-Prevenzione Investigators. Dietary supplementation with n-3 polyunsaturated fatty acids and vitamin $E$ in 11,324 patients with myocardial infarction: results of the GISSI-Prevenzione trial. Lancet 1999; 354: 447455

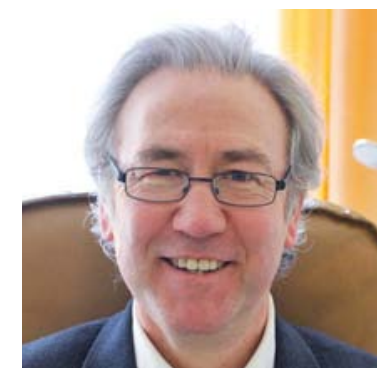

\section{Dr. med. Vollker Schmiedel}

Paramed

Haldenstr. 1

\section{CH-6340 Baar}

E-Mail: v.schmiedel@paramed.ch

Volker Schmiedel war von 1996-2015 Chefarzt der Inneren Abteilung der Habichtswaldklinik (Kassel) und ist seit Oktober 2015 Arzt im ganzheitlichen Ambulatorium Paramed in Baar (Schweiz). Er ist zudem Fortbildungsleiter für „Naturheilverfahren“ der Medizinischen Woche, Mitherausgeber der Zeitschrift „Erfahrungsheilkunde“ und des „Leitfaden Naturheilkunde“ sowie Autor zahlreicher weiterer naturheilkundlicher Bücher für Therapeuten und Laien. 